\title{
A GENERAL LOWER BOUND FOR POTENTIALLY $H$-GRAPHIC SEQUENCES
}

\author{
MICHAEL J. FERRARA \\ DEPARTMENT OF THEORETICAL AND APPLIED MATHEMATICS \\ THE UNIVERSITY OF AKRON \\ MJF@UAKRON.EDU \\ JOHN SCHMITT \\ DEPARTMENT OF MATHEMATICS \\ MIDDLEBURY COLLEGE \\ JSCHMITT@MIDDLEBURY.EDU
}

\begin{abstract}
We consider a variation of the classical Turán-type extremal problem as introduced by Erdős et al. in [7]. Let $\pi$ be an $n$-element graphic sequence, and $\sigma(\pi)$ be the sum of the terms in $\pi$, that is the degree sum. Let $H$ be a graph. We wish to determine the smallest $m$ such that any $n$-term graphic sequence $\pi$ having $\sigma(\pi) \geq m$ has some realization containing $H$ as a subgraph. Denote this value $m$ by $\sigma(H, n)$. For an arbitrarily chosen $H$, we construct a graphic sequence $\pi^{*}(H, n)$ such that $\sigma\left(\pi^{*}(H, n)\right)+2 \leq \sigma(H, n)$. Furthermore, we conjecture that equality holds in general, as this is the case for all choices of $H$ where $\sigma(H, n)$ is currently known. We support this conjecture by examining those graphs that are the complement of triangle-free graphs, and showing that the conjecture holds despite the wide variety of structure in this class. We will conclude with a brief discussion of a connection between potentially $H$-graphic sequences and $H$-saturated graphs of minimum size.

Keywords: Degree sequence, Potentially graphic sequence, $H$-saturated graph.
\end{abstract}

\section{INTRODUCTION}

A good reference for any undefined terms is [1]. Let $G$ be a simple undirected graph, without loops or multiple edges. Let $V(G)$ and $E(G)$ denote the vertex set and edge set of $G$ respectively and let $d(v)$ denote the degree of a vertex $v$. Let $\bar{G}$ denote the complement of $G$. Denote the complete graph on $t$ vertices and the complete bipartite graph with partite sets of size $r$ and $s$ by $K_{t}$ and $K_{r, s}$, respectively. Additionally, let $K_{s}^{t}$ denote the complete balanced multipartite graph with $t$ partite sets of size $s$. Given any two graphs $G$ and $H$, their join, denoted $G+H$, is the graph with $V(G+H)=V(G) \cup V(H)$ and $E(G+H)=E(G) \cup$ $E(H) \cup\{g h \mid g \in V(G), h \in V(H)\}$. Additionally, let $\alpha(G)$ denote the independence number of $G$. If $H$ is a subgraph of $G$, we will write $H \subset G$, and if $H$ is an induced subgraph of $G$, we will write $H<G$.

A sequence of nonnegative integers $\pi=\left(d_{1}, d_{2}, \ldots, d_{n}\right)$ is called graphic if there is a (simple) graph $G$ of order $n$ having degree sequence $\pi$. In this case, $G$ is said 
to realize $\pi$, and we will write $\pi=\pi(G)$. If a sequence $\pi$ consists of the terms $d_{1}, \ldots, d_{t}$ having multiplicities $\mu_{1}, \ldots, \mu_{t}$, we may write $\pi=\left(d_{1}^{\mu_{1}}, \ldots, d_{t}^{\mu_{t}}\right)$.

For a given graph $H$, a sequence $\pi$ is said to be potentially H-graphic if there is some realization of $\pi$ which contains $H$ as a subgraph. Additionally, let $\sigma(\pi)$ denote the sum of the terms of $\pi$. Define $\sigma(H, n)$ to be the smallest integer $m$ so that every $n$-term graphic sequence $\pi$ with $\sigma(\pi) \geq m$ is potentially $H$-graphic. In this paper, given an arbitrary $H$, we construct a graphic sequence $\pi^{*}(H, n)$ such that $\sigma\left(\pi^{*}(H, n)\right)+2 \leq \sigma(H, n)$. We then show that equality holds for all graphs $H$ that are the complement of a triangle-free graph. There have been numerous papers, including but certainly not limited to [5], [3], [4], [7], [9], [11], [12], [14], [15], [16], [17] and [18], that consider the potential problem for specific graphs or narrow families of graphs. It is our hope that the ideas and results presented in this paper will facilitate a broader consideration of problems of this type.

\section{A Short History}

In this section, we present the extremal sequences for two classes of graphs: complete graphs and complete balanced bipartite graphs. Our goal is to motivate the general constructions in the next section.

2.1. $H=K_{t}$. In [7] Erdös, Jacobson and Lehel conjectured that $\sigma\left(K_{t}, n\right)=(t-$ $2)(2 n-t+1)+2$. The conjecture rises from consideration of the graph $K_{(t-2)}+$ $\bar{K}_{(n-t+2)}$. It is easy to observe that this graph contains no $K_{t}$, is the unique realization of the sequence $\left((n-1)^{t-2},(t-2)^{n-t+2}\right)$, and has degree sum $(t-$ $2)(2 n-t+1)$. The cases $t=3,4$ and 5 were proved separately (see respectively [7], [12] and [15], and [16]), and Li, Song and Luo [17] proved the conjecture true via linear algebraic techniques for $t \geq 6$ and $n \geq\left(\begin{array}{l}t \\ 2\end{array}\right)+3$. A purely graph-theoretic proof was given in [10] and also as a corollary to the main result in [4].

2.2. $H=K_{s, s}$. The following results appears in [12] and [18]. Here $E_{1}, E_{2}, E_{3}$ and $E_{4}$ are somewhat technical numerical classes which, based on the parity of $n$ and $s$, assure that the given degree sums are even.

Theorem 2.1. - If $s$ is an odd, positive integer and $n \geq 4 s^{2}+3 s-8$, then

$$
\sigma\left(K_{s, s}, n\right)= \begin{cases}\left(\frac{5}{2} s-\frac{5}{2}\right) n-\frac{11}{8} s^{2}+\frac{5}{2} s+\frac{7}{8} & \text { if }(s, n) \in E_{3} \\ \left(\frac{5}{2} s-\frac{5}{2}\right) n-\frac{11}{8} s^{2}+\frac{5}{2} s+\frac{15}{8} & \text { if }(s, n) \in E_{4} .\end{cases}
$$

- If $s$ is an even, positive integer and $n \geq 4 s^{2}-s-6$, then

$$
\sigma\left(K_{s, s}, n\right)= \begin{cases}\left(\frac{5}{2} s-2\right) n-\frac{11}{8} s^{2}+\frac{5}{4} s+2 & \text { if }(s, n) \in E_{1} \\ \left(\frac{5}{2} s-2\right) n-\frac{11}{8} s^{2}+\frac{5}{4} s+1 & \text { if }(s, n) \in E_{2} .\end{cases}
$$

In order to establish a lower bound on $\sigma\left(K_{s, s}, n\right)$ the authors present several sequences dependent on the parities of $s$ and $n$. 
(i) If $s$ is odd and $(s, n) \in E_{3}$, then

$$
\begin{aligned}
\pi\left(K_{s, s}, n\right)=\left((n-1)^{s-1}, 2 s-2,2 s-\right. & 3, \ldots, \frac{3}{2} s+\frac{3}{2}, \frac{3}{2} s+\frac{1}{2}, \\
& \left.\left(\frac{3}{2} s-\frac{1}{2}\right)^{\frac{s}{2}+\frac{3}{2}},\left(\frac{3}{2} s-\frac{3}{2}\right)^{n-2 s}, \frac{3}{2} s-\frac{5}{2}\right) .
\end{aligned}
$$

(ii) If $s$ is odd and $(s, n) \in E_{4}$, then

$$
\begin{aligned}
\pi\left(K_{s, s}, n\right)=\left((n-1)^{s-1}, 2 s-2,2 s-3, \ldots\right. & , \frac{3}{2} s+\frac{3}{2}, \frac{3}{2} s+\frac{1}{2}, \\
& \left.\left(\frac{3}{2} s-\frac{1}{2}\right)^{\frac{s}{2}+\frac{3}{2}},\left(\frac{3}{2} s-\frac{3}{2}\right)^{n-2 s+1}\right) .
\end{aligned}
$$

(iii) If $s$ is even and $(s, n) \in E_{1}$, then

$$
\pi\left(K_{s, s}, n\right)=\left((n-1)^{s-1}, 2 s-2,2 s-3, \ldots, \frac{3}{2} s+1, \frac{3}{2} s,\left(\frac{3}{2} s-1\right)^{\left.n-\frac{3}{2} s+2\right)}\right) .
$$

(iv) If $s$ is even and $(s, n) \in E_{2}$, then

$$
\pi\left(K_{s, s}, n\right)=\left((n-1)^{s-1}, 2 s-2,2 s-3, \ldots, \frac{3}{2} s+1, \frac{3}{2} s,\left(\frac{3}{2} s-1\right)^{n-\frac{3}{2} s+1},\left(\frac{3}{2} s-2\right)\right) .
$$

Each of these sequences can be realized by the join of $K_{s-1}$ and some graph $H^{\prime}$. This $H^{\prime}$ has no vertices of degree $s$, one vertex of degree $s-1$, two vertices of degree $s-2$ and so on. More generally, no choice of $H^{\prime}$ contains $x_{1}$ vertices of degree $x_{2}$, where $x_{1}+x_{2}=s+1$. This implies that $H^{\prime}$ cannot possibly contain a copy of $K_{x_{1}, x_{2}}$. However, if any of these sequences were to be potentially $K_{s, s}$ graphic, at least $s+1$ of the vertices in a copy of $K_{s, s}$ would have to be chosen from $H^{\prime}$. These vertices in turn, would comprise some $K_{x_{1}, x_{2}}$ where $x_{1}+x_{2}=s+1$.

\section{A General Lower Bound}

We assume that $H$ has no isolated vertices and furthermore that $n$ is sufficiently large relative to $|V(H)|$. We define the quantities

$$
u(H)=|V(H)|-\alpha(H)-1,
$$

and

$$
d(H)=\min \{\Delta(F): F<H,|V(F)|=\alpha(H)+1\} .
$$

Consider the following sequence,

$$
\widehat{\pi}(H, n)=\left((n-1)^{u(H)},(u(H)+d(H)-1)^{n-u(H)}\right) .
$$

If this sequence is not graphic, that is if $n-u(H)$ and $d(H)-1$ are both odd, we reduce the smallest term by one. To see that this will result in a graphic sequence, we make two observations. First, $(d(h)-1)$-regular graphs of order $n-u(H) \geq d(H)$ exist whenever $d(H)-1$ and $n-u(H)$ are not both odd. If $n$ and $d(H)-1$ are both odd, it is not difficult to show that the sequence $\left((d(H)-1)^{n-u(H)-1}, d(H)-2\right)$ is graphic

Every realization of $\widehat{\pi}(H, n)$ is a complete graph on $u(H)$ vertices, joined to a graph, call it $G^{\prime}$, that is either $(d(H)-1)$-regular or nearly so. Note that the 
subgraph induced by any $\alpha(H)+1$ vertices of $H$ has maximum degree at least $d(H)$. Thus, no realization of $\widehat{\pi}(H, n)$ could possibly contain a copy of $H$, as at least $\alpha(H)+1$ vertices of such a subgraph would have to lie in $G^{\prime}$.

The degree sum of (7) is

$$
\sigma(\widehat{\pi}(H, n))=n(2 u(H)+d(H)-1)-u(H)(u(H)+d(H)),
$$

and if both $n-u(H)$ and $d(H)-1$ are odd, the sum will be one smaller.

To gain some additional insight, we will consider first the case $H=K_{t}$. Then $u\left(K_{t}\right)=t-2$ and $d\left(K_{t}\right)=1$, so that

$$
\widehat{\pi}\left(K_{t}, n\right)=\left((n-1)^{t-2},(t-2)^{n-t+2}\right) .
$$

This is exactly the extremal sequence put forth to establish the lower bound for $\sigma\left(K_{t}, n\right)$. Similarly, the extremal sequences used to determine $\sigma\left(k K_{2}, n\right), \sigma\left(C_{2 k+1}, n\right)$ and $\sigma\left(K_{1}+k K_{2}, n\right)$ are precisely $\widehat{\pi}\left(k K_{2}, n\right), \widehat{\pi}\left(C_{2 k+1}, n\right)$ and $\widehat{\pi}\left(K_{1}+k K_{2}, n\right)$, respectively (see [12],[14] and[11]). However, $\sigma\left(\widehat{\pi}\left(K_{s, s}, n\right)\right)$ is asymptotically equivalent to, but smaller than $\sigma\left(K_{s, s}, n\right)$. Along these lines, we are able to refine the sequence given above.

For convenience, let $d=d(H), u=u(H)$ and $\alpha=\alpha(H)$ and let $v_{i}(H)$ denote the number of vertices of degree $i$ in $H$. For all $i, d \leq i \leq \alpha$ we define the quantity $m_{i}$ to be the minimum number of vertices of degree at least $i$ over all induced subgraphs $F$ of $H$ with $|V(F)|=\alpha+1$ and $\sum_{j=i}^{\alpha} v_{j}(F)>0$ and 0 if no such subgraphs exist. The quantities $n_{i}, d \leq i \leq \alpha$, are defined recursively such that $n_{d}=m_{d}-1$ and either $n_{i}=\min \left\{m_{i}-1, n_{i-1}\right\}$ if $m_{i} \geq 1$ or $n_{i}=0$ if $m_{i}=0$. Finally, we define $\delta_{\alpha-1}=n_{\alpha-1}$ and for $d \leq i \leq \alpha-2$ we define $\delta_{i}=n_{i}-n_{i+1}$. We do not define $\delta_{\alpha}$, as any induced subgraph composed of a maximum independent set and an additional vertex has at most one vertex of degree $\alpha$, and as such $n_{\alpha}$ is always 0 .

We now consider the following sequence:

$\pi^{*}(H, n)=\left((n-1)^{u},(u+\alpha-1)^{\delta_{\alpha-1}},(u+\alpha-2)^{\delta_{\alpha-2}}, \ldots(u+d)^{\delta_{d}},(u+d-1)^{n-u-\Sigma \delta_{i}}\right)$.

The sequence $\pi^{*}$ is constructed so that it contains $n_{i}$ terms that are at least $u+i$ and $\delta_{i}$ terms that are exactly $u_{i}$.

If this sequence is not graphic, then we will reduce the smallest term which is strictly greater than $u(H)$ in the sequence by one and redefine $\pi^{*}(H, n)$ to be this graphic sequence instead. The following is the main result of this paper.

Theorem 3.1. Given a graph $H$, with $u(H)$ and $d(H)$ as above, and $n$ sufficiently large then,

$$
\sigma(H, n) \geq \max \left\{\sigma\left(\pi^{*}\left(H^{*}, n\right)\right)+2 \mid H^{*} \subseteq H\right\} .
$$

Proof. Let $H^{*}$ be the subgraph of $H$ that realizes the maximum above. Let $G$ be any realization of $\pi^{*}\left(H^{*}, n\right)$. We show that $G$ does not contain a copy of $H^{*}$. 
Note that this degree sequence implies that $G$ is a copy of $K_{u\left(H^{*}\right)}$ joined to another graph $G^{*}$ on $n-u\left(H^{*}\right)$ vertices. Assume that there is a copy of $H^{*}$ contained in $G$. There are at least $\alpha\left(H^{*}\right)+1$ vertices from $G^{*}$ that must belong to this copy of $H$. Let $H^{* *}$ denote the subgraph of $H^{*}$ induced by these $\alpha\left(H^{*}\right)+1$ vertices. Notice, however, no $\alpha\left(H^{*}\right)+1$ vertices of $G^{*}$ have sufficient degree to contain a copy of any $H^{* *}$. In particular, if $\sum_{j \geq \ell} v_{j}\left(H^{* *}\right)>0$ then $H^{* *}$ contains at least $m_{\ell}$ vertices of degree $\ell$ or greater. By our construction, there are at most $n_{\ell} \leq m_{\ell}-1$ vertices of degree at least $\ell$ in $G^{*}$. This contradicts the assumption that $H^{* *} \subseteq G^{*}$. Thus, $G$ contains no copy $H^{*}$ and hence no copy of $H$.

Theorem 3.1 requires that we examine all subgraphs of $H$. To see that this is necessary, we consider the split graph $K_{t}+\overline{K_{s}}$ with a pendant vertex $v$ adjacent to one of the vertices in the independent set of order $s$. For this choice of $H$, $\alpha(H)=s$ and hence $u(H)=(s+t+1)-s-1=t$ and $d(H)=1$. However, if we remove $v$, the pendant vertex, and consider the split graph, we can see that $u\left(K_{t}+\overline{K_{s}}\right)=t-1$ but any $s+1$-vertex subgraph of $K_{t}+\overline{K_{s}}$ must contain some vertex from the $K_{t}$, implying that $d\left(K_{t}+\overline{K_{s}}\right)=s$. Therefore, if we choose $s \geq 3, \sigma\left(\pi^{*}\left(K_{t}+\overline{K_{s}}, n\right)\right) \geq \sigma\left(\pi^{*}(H, n)\right)$.

The reader should note that for any values of $n$ and $s, \pi^{*}\left(K_{s, s}, n\right)$ is exactly those sequences given in (3)-(6). Additionally, given values of $n, s$ and $t, \pi^{*}\left(K_{s}^{t}, n\right)$ matches the extremal sequences given in [23].

We conjecture that equality holds in Theorem 3.1.

Conjecture 1. Let $H$ be any graph, and let $n$ be a sufficiently large integer. Then

$$
\sigma(H, n)=\max \left\{\sigma\left(\pi^{*}\left(H^{*}, n\right)\right)+2 \mid H^{*} \subseteq H\right\} .
$$

We also pose the weaker conjecture, that the bound put forth is asymptotically correct.

Conjecture 2. Let $H$ be any graph, and let $\epsilon>0$. Then there exists an $n_{0}=$ $n_{0}(\epsilon, H)$ such that for any $n>n_{0}$

$$
\sigma(H, n) \leq \max \left\{\left(n\left(2 u\left(H^{*}\right)+d\left(H^{*}\right)-1+\epsilon\right) \mid H^{*} \subseteq H\right\}\right.
$$

Conjectures 1 and 2 have been verified for a wide variety of graphs. This includes, but is not limited to: complete graphs and unions of complete graphs [7], [9], [12], [15], [16], [17], complete bipartite graphs [3],[12], [18], complete multipartite graphs [5], [20], matchings [12], cycles [14], (generalized) friendship graphs [2], [9], [11], and split graphs [4] At this time we know of no subgraph for which these conjectures do not hold for sufficiently large $n$.

While Conjecture 1 seems challenging, we feel that there is a good chance that Conjecture 2 could be verified. In the following section, we will verify Conjecture 1 for a broad class of graphs. 


\section{Complements of Triangle-Free Graphs}

We now turn our attention to graphs $H$ of order $k \geq 3$ with $\alpha(H)=2$, or those graphs that are the complement of a triangle-free graph. The main result of this section is as follows.

Theorem 4.1. Let $H$ be any graph of order $k$ with $\alpha(H)=2$. Then

$$
\sigma(H, n)=\sigma\left(\pi^{*}(H, n)\right)+2 .
$$

Any graph $H$ in this class has $u(H)=k-3$ and $d(H) \leq 2$. We prove Theorem 4.1 by considering the cases $d(H)=1$ and $d(H)=2$ separately. In each case we construct a graph $H(d)$ that contains $H$ as a subgraph and show that $\sigma(H(d), n)=$ $\sigma\left(\pi^{*}(H, n)\right)+2$. This implies that $\max \left\{\sigma\left(\pi^{*}\left(H^{*}, n\right)\right)+2 \mid H^{*} \subseteq H\right\}=\sigma\left(\pi^{*}(H, n)\right)+$ 2 .

The following result from [4] will be very useful.

Theorem 4.2. If $n \geq 3 s+2 t^{2}+3 t-3$ then

$\sigma\left(K_{s}+\bar{K}_{t}, n\right)=\left\{\begin{array}{l}(t+2 s-3) n-(s-1)(s+t-1)+2 \quad \text { if } t \text { or } n-s \text { is odd. } \\ (t+2 s-3) n-(s-1)(s+t-1)+1 \quad \text { if } t \text { and } n-s \text { are even. }\end{array}\right.$

It is not difficult to see that if $d(H)=2$ then $H$ is isomorphic to $K_{k}-t K_{2}$, where $k$ is the order of $H$ and $t$ is some positive integer that is at most $\frac{k}{2}$. Let $H$ be a graph of order $k \geq 3$ with $\alpha(H)=2$ and $d(H)=2$ and let $n \geq k$ be an integer. Then, by (9), we have.

(i) If $n \equiv k-3(\bmod 2)$ then

$$
\pi^{*}(H, n)=\left((n-1)^{k-3},(k-2)^{n-k+3}\right)
$$

(ii) If $n \not \equiv k-3(\bmod 2)$ then

$$
\pi^{*}(H, n)=\left((n-1)^{k-3},(k-2)^{n-k+2}, k-3\right)
$$

Proposition 4.3. Let $H$ be a graph of order $k$ with $\alpha(H)=2$ and $d(H)=2$, and let $n$ be a sufficiently large integer. Then

$$
\sigma(H, n)=\sigma\left(\pi^{*}(H, n)\right)+2=n(2 k-5)-k^{2}+4 k-1-m,
$$

where $m=n-k+3(\bmod 2)$.

Proof. The fact that $\sigma(H, n) \geq \sigma\left(\pi^{*}(H, n)\right)+2$ follows from Theorem 3.1. Note that any $H$ with $\alpha(H)=2$ and $d(H)=2$ is a subgraph of $K_{k-2}+\bar{K}_{2}$ so that $\sigma(H, n) \leq \sigma\left(K_{k-2}+\bar{K}_{2}, n\right)$. Theorem 4.2 implies

$$
\sigma\left(K_{k-2}+\bar{K}_{2}, n\right)=n(2 k-5)-k^{2}+4 k-1+m=\sigma\left(\pi^{*}(H, n)\right)+2 .
$$

The proposition follows.

Those graphs $H$ with $\alpha(H)=2$ and $d(H)=1$ have a considerably wider variety of structures. Any graph $H$ in this class is the complement of a triangle-free graph $G$ that is not a matching. The disjoint union of two cliques falls into this class, as does $K_{k}-t P_{3}$ and many other graphs of varying densities. We are able to verify Conjecture 1 for this diverse class of graphs. Our first observation is that any graph 
$H$ with $\alpha(H)=2$ and $d(H)=1$ must contain $K_{2} \cup K_{1}$ as an induced subgraph, as this is the only graph on 3 vertices with maximum degree 1 . This also immediately implies that $m_{d}=m_{1}=2$. Therefore, if $H$ is any graph of order $\mathrm{k}$ with $\alpha(H)=2$ and $d(H)=1$ and $n \geq k$ is an integer, then (9) implies that

$$
\pi^{*}(H, n)=\left((n-1)^{k-3},(k-3)^{n-k+3}\right) .
$$

The following lemma from [12] will be useful in the next proof.

Lemma 4.4. If $\pi$ is a graphical sequence with a realization $G$ containing $H$ as a subgraph, then there is a realization $G^{\prime}$ of $\pi$ containing $H$ with the vertices of $H$ having the $|V(H)|$ largest degrees of $\pi$.

We now show that Conjecture 1 holds when $\alpha(H)=2$ and $d(H)=1$.

Proposition 4.5. Let $H$ be a graph of order $k$ with $\alpha(H)=2$ and $d(H)=1$, and let $n$ be a sufficiently large integer. Then

$$
\sigma(H, n)=\sigma\left(\pi^{*}(H, n)\right)+2=n(2 k-6)-k^{2}+5 k-4
$$

Proof. Let $\pi$ be a nonincreasing, $n$-term graphic sequence with $\sigma(\pi) \geq n(2 k-6)-$ $k^{2}+5 k-4$. Note that if $n$ is sufficiently large, $\sigma(\pi) \geq \sigma\left(K_{k-1}, n\right) \geq \sigma\left(K_{k-3}+\bar{K}_{3}, n\right)$. We will show that $\pi$ has a realization containing $K_{k-3}+\left(K_{2} \cup K_{1}\right)$ and, as we have previously observed that $H$ must contain an induced copy of $K_{2} \cup K_{1}$, a copy of $H$.

Let $G$ be a realization of $\pi$ that contains a copy of $K_{k-3}+\bar{K}_{3}$ on the $k$ vertices of highest degree in $G$. Such a realization exists by Lemma 4.4. Let $S$ denote this subgraph, $F$ denote the complete subgraph of order $k-3$ and let $I$ denote the independent set of order 3 in $S$, so that $S=F+I$. We can assume that $F$ is comprised of the $k-3$ vertices of highest degree in $G$. If not, there are vertices $x$ in $I$ and $y$ in $F$ such that $d(y)<d(x)$. We wish to create a realization of $G$ containing a copy of $K_{k-3}+\bar{K}_{3}$ on the $k$ vertices of highest degree such that $x$ is in $F$ and $y$ is in $I$. If $x$ is adjacent to all the other vertices in $S$, we can simply exchange the roles of $x$ and $y$. If $x$ was not adjacent to exactly one vertex in $I$, say $v$, then as $d(x)>d(y)$ there is some vertex $w$ outside of $S$ that is adjacent to $x$ but not to $y$. We will create a new realization of $\pi$ by adding the edges $y w$ and $x v$ and deleting the edges $y v$ and $x w$. The case where $x$ is not adjacent to exactly two vertices in $I$ is handled similarly. Repeating this process allows us to create a realization of $\pi$ containing $K_{k-3}+\bar{K}_{3}=F+I$ in which the $k-3$ highest degree vertices of $G$ lie in $F$.

Let $x_{1}$ and $x_{2}$ be the vertices in $I$ having the highest degrees, and note that $\sigma(\pi) \geq \sigma\left(K_{k-1}, n\right)$ implies $d\left(x_{1}\right)$ and $d\left(x_{2}\right)$ are both at least $k-2$. If there is any edge in the subgraph induced by $I$, then $G$ contains a copy of $K_{k-3}+\left(K_{2} \cup K_{1}\right)$ and we are done. Therefore, we may assume that $I$ is an independent set. Let $N_{1}$ and $N_{2}$ denote $N\left(x_{1}\right) \backslash S$ and $N\left(x_{2}\right) \backslash S$, respectively, and note that both of these sets are nonempty since $d\left(x_{1}\right)$ and $d\left(x_{2}\right)$ are both at least $k-2$. If $y_{1}$ and $y_{2}$ are distinct vertices in $N_{1}$ and $N_{2}$, respectively, then we may assume that $y_{1}$ and $y_{2}$ are adjacent. If they are not, then we would exchange the edges $x_{1} y_{1}$ and $x_{2} y_{2}$ for the nonedges $x_{1} x_{2}$ and $y_{1} y_{2}$, creating an edge in $I$ and completing the proof. 
The goal of the next part of this proof is to show that we may assume that there is some vertex $v$ in $F$ such that $d(v) \leq 4 k$.

Consider first the case where $N_{2} \subseteq N_{1}\left(N_{1} \subseteq N_{2}\right.$ is handled identically) and let $w$ be a vertex in $N_{2}$. If $\left|N_{1} \backslash N_{2}\right|>k$ then $d(w)>d\left(x_{2}\right)$ since $w$ is adjacent to every vertex in $N_{1} \backslash N_{2}$. We therefore assume that $\left|N_{1} \backslash N_{2}\right| \leq k$. Also note that $N_{1} \cap N_{2}$ is a clique, and hence contains at most $k-2$ vertices. There is some vertex $v$ in $F$ that is not adjacent to $w$, otherwise $d(w)>d\left(x_{1}\right)$, which contradicts our choice of $G$. Let $y$ be a neighbor of $v$ that does not lie in $S \cup N_{1} \cup N_{2}$. If no such $y$ exists, then clearly $d(v) \leq 4 k$. We claim that $w y$ is an edge of $G$, lest we could exchange the edges $x_{1} w, x_{2} w$ and $y v$ for the nonedges $w v, w y$ and $x_{1} x_{2}$ (see Figure 1 ), creating an edge in $I$. However, if the degree of $v$ is more than $4 k$ there are at least $k-1$ such choices for $y$. This implies that $d(w) \geq k+\left|N_{1}\right|>d\left(x_{1}\right)$, which contradicts our choice of $G$. Thus we may assume that $d(v) \leq 4 k$.

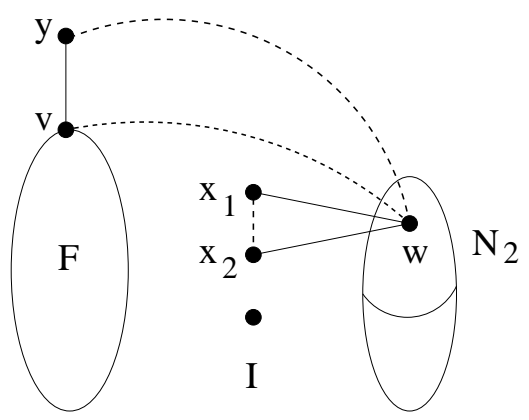

Figure 1. $N_{2} \subseteq N_{1}$

Assume now that there is some vertex $w_{1}$ in $N_{1} \backslash N_{2}$ and some vertex $w_{2}$ in $N_{2} \backslash N_{1}$. We first show that $N_{1} \cup N_{2}$ is complete. To accomplish this, we need only show that for any $w_{1}^{\prime}$ in $N_{1} \backslash N_{2}, w_{1} w_{1}^{\prime}$ is an edge of $G$ (or symmetrically, if $w_{2}^{\prime}$ is an element of $N_{2} \backslash N_{1}$ then $w_{2} w_{2}^{\prime}$ is an edge in $G$ ). If not, we can exchange the edges $x_{1} w_{1}, x_{1} w_{1}^{\prime}$ and $x_{2} w_{2}$ for the nonedges $w_{1} w_{1}^{\prime}, x_{1} w_{2}$ and $x_{1} x_{2}$, creating an edge in $I$ and completing the proof. Thus, since $N_{1} \cup N_{2}$ is complete we may assume that $\left|N_{1} \cup N_{2}\right| \leq k-1$. Again, there is some $v$ in $F$ such that $w_{2}$ is not adjacent to $v$, lest $d\left(w_{2}\right)>d\left(x_{2}\right)$. Let $y$ be any neighbor of $v$ not in $S \cup N_{1} \cup N_{2}$. Then $w_{1}$ is adjacent to $y$ or else we could exchange the edges $y v, x_{1} w_{1}$ and $x_{2} w_{2}$ for the nonedges $y w_{1}, v w_{2}$ and $x_{1} x_{2}$ (see Figure 2), creating an edge in $I$. If $d(v)>3 k$, then there are at least $k$ such choices for $y$, implying that $d\left(w_{1}\right) \geq k+\left|N_{1} \cup N_{2}\right|-1>d\left(x_{1}\right)$, a contradiction.

Hence, we may assume that there is some vertex $v$ in $F$ such that $d(v) \leq 4 k$. As a result, there are at most $(k-4)(n-1)+4 k$ edges adjacent to vertices in $F$, at most $12 k$ edges adjacent to vertices in $I$ and, as both $N_{1}$ and $N_{2}$ have at most $4 k$ vertices each, at most $4 k(8 k)=32 k^{2}$ edges adjacent to vertices in $N_{1} \cup N_{2}$. This is at most $(k-4) n+32 k^{2}+15 k+4$ edges. However, there are at least $\sigma(\pi) / 2=(k-3+o(1)) n$ edges in $G$, so for $n$ sufficiently large there is some edge $y z$ in $G$ such that $y$ is not adjacent to any $w_{1}$ in $N_{1}$ and $z$ is not adjacent to any $w_{2}$ in $N_{2}$, where $w_{1}$ and $w_{@}$ may be the same vertex. We can therefore exchange the edges $x_{1} w_{1}, x_{2} w_{2}$ and 


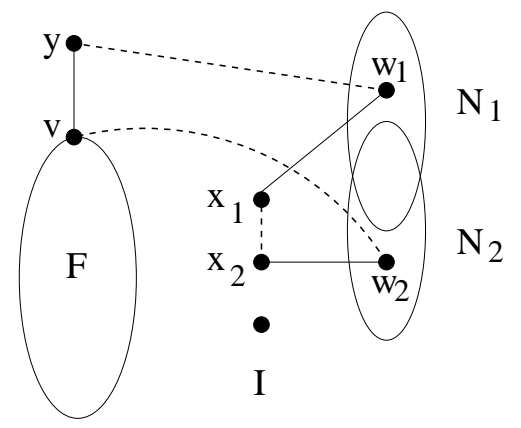

Figure 2. $N_{2} \nsubseteq N_{1}$ and $N_{1} \nsubseteq N_{2}$

$y z$ for the nonedges $w_{1} y, w_{2} z$ and $x_{1} x_{2}$, creating an edge in $I$, and completing the proof.

Propositions 4.3 and 4.5 together imply Theorem 4.1. As mentioned above, there is quite a wide variety to the structures of those graphs $H$ having independence number 2 , and yet we have demonstrated that $\sigma(H, n)$ for this class depends only on the value of $d(H)$, as suggested by Conjecture 1 .

\section{H-Saturated Graphs}

Here we describe the relationship of $\sigma(H, n)$ to another extremal function $\operatorname{sat}(n, H)$. We begin with the relevant terminology and results.

A graph $G$ is said to be $H$-saturated if $G$ contains no copy of $H$ as a subgraph and for any edge $e$ not in $G, G+e$ does contain a copy of $H$. The problem of determining the minimum number of edges in an $H$-saturated graph, denoted $\operatorname{sat}(n, H)$, was first considered in 1963 by Erdős, Hajnal and Moon [6] for $H=K_{t}$. They determined that $\operatorname{sat}\left(n, K_{t}\right)=(t-2)(n-1)-\left(\begin{array}{c}t-2 \\ 2\end{array}\right)$, which arises from consideration of the split graph $K_{t-2}+\bar{K}_{n-t+2}$. The best known upper bound for an arbitrary graph $H$ is given by the following result of Kászonyi and Tuza [13].

Theorem $5.1([13])$. Let $u(H)$ be as defined above, and set

$$
s(H)=\min \left\{e\left(H^{*}\right)\left|\alpha\left(H^{*}\right)=\alpha(H),\right| V\left(H^{*}\right) \mid=\alpha(H)+1, H^{*} \subseteq H\right\}
$$

then,

$$
\operatorname{sat}(n, H) \leq n\left(u(H)+\frac{s(H)-1}{2}\right)-\frac{u(H)(u(H)+s(H))}{2} .
$$

The reader should note that the bound given in Theorem 5.1 reflects the number of edges in the join of $K_{u(H)}$ and a graph which is (nearly) $(s-1)$-regular. Comparing Theorem 5.1 to the construction of $\pi^{*}(H, n)$, we note that $d(H) \leq s(H)$ and hence that if $i \geq s(H), n_{i}=0$. Theorem 5.1 and Theorem 3.1 immediately imply the following result. 
Theorem 5.2. Given a graph $H$, if there exists an $H^{\prime} \subseteq H$ with $2 u\left(H^{\prime}\right)+d\left(H^{\prime}\right)-$ $1 \geq 2 u(H)+s(H)-1$ then for $n$ sufficiently large we have

$$
2 \operatorname{sat}(n, H)<\sigma(H, n) \text {. }
$$

In particular, this result holds if $d(H)=s(H)$.

We strongly believe that the conclusion of Theorem 5.2 holds in general, even though the hypothesis does not. Therefore, we conjecture the following.

Conjecture 3. Let $H$ be a graph and let $n$ be a sufficiently large integer. Then

$$
2 \operatorname{sat}(n, H)<\sigma(H, n) \text {. }
$$

As the problem of determining $\operatorname{sat}(n, H)$ has proven difficult over time, we are not able to confirm Conjecture 3 in as many cases as Conjectures 1 and 2 . We know that Conjecture 3 holds for complete graphs [6], [7], $t K_{p}$ and certain generalized friendship graphs [8], $C_{4}[12],[22],[24]$, and $K_{1, t}[13]$.

\section{Conclusion}

In light of Theorem 4.1, it may be interesting to individually consider classes of graphs with fixed independence number. This may be a fruitful direction, although the diversity in the structures of the $\alpha(H)+1$ vertex induced subgraphs of such graphs rapidly increases. We feel that this line of investigation would move us closer to the goal of verifying either of Conjectures 1 and 2 .

The authors would like to thank Mike Jacobson for his helpful comments and insightful questions that led to Theorem 4.1.

\section{REFERENCES}

[1] B. Bollobás, Extremal Graph Theory, Academic Press Inc. (1978).

[2] G. Chen, J. Schmitt, J.H. Yin, Graphic Sequences with a Realization Containing a Generalized Friendship Graph to appear in Discrete Mathematics

[3] G. Chen, J. Li, J. Yin, A variation of a classical Turán-type extremal problem. European Journal of Combinatorics 25 (2004) 989-1002.

[4] G. Chen, J. Yin, On Potentially $K_{r_{1}, r_{2}, \ldots, r_{m}}$-graphic Sequences, preprint.

[5] Guantao Chen, M. Ferrara, R. Gould, J. Schmitt, Graphic Sequences with a Realization Containing a Complete Multipartite Subgraph, to appear in Discrete Mathematics

[6] P. Erdős, A. Hajnal, J.W. Moon, A problem in graph theory. Amer. Math. Monthly 71 (1964) 1107-1110.

[7] P. Erdős, M.S. Jacobson, J. Lehel, Graphs Realizing the Same Degree Sequence and their Respective Clique Numbers. Graph Theory, Combinatorics and Applications, Vol. I, 1991, ed. Alavi, Chartrand, Oellerman and Schwenk, 439-449.

[8] R. Faudree, M. Ferrara, R. Gould, M. Jacobson, $t K_{p}$-saturated graphs, submitted.

[9] M. Ferrara, Graphic Sequences with a Realization Containing a Union of Cliques, Graphs and Combinatorics 23 (2007), 263-269.

[10] M. Ferrara, R. Gould and J. Schmitt, Using Edge Swaps to Prove the Erdos-JacobsonLehel Conjecture, to appear in Bulletin of the ICA. 
[11] M. Ferrara, R. Gould and J. Schmitt, Graphic Sequences with a Realizaton Containing a Friendship Graph, ARS Combinatoria 85 (2007), 161-171.

[12] R. Gould, M. Jacobson, J. Lehel, Potentially G-graphic degree sequences. Combinatorics, Graph Theory, and Algorithms (eds. Alavi, Lick and Schwenk), Vol. I, New York: Wiley \& Sons, Inc., 1999, 387-400.

[13] L. Kászonyi, Z. Tuza, Saturated graphs with minimal number of edges. J. Graph Theory 10 (1986) 203-210.

[14] C. Lai, The smallest degree sum that yields potentially $C_{k}$-graphical sequences. $J$. Combin. Math. Combin. Comput. 49 (2004), 57-64.

[15] J. Li, Z. Song, An extremal problem on the potentially $P_{k}$-graphic sequences. The International Symposium on Combinatorics and Applications, June 28-30, 1996 (W.Y.C. Chen et. al., eds.) Tanjin, Nankai University 1996, 269-276.

[16] J. Li, Z. Song, The smallest degree sum that yields potentially $P_{k}$-graphical sequences. J. Graph Theory 29 (1998), no.2, 63-72.

[17] J. Li, Z. Song, R. Luo, The Erdős-Jacobson-Lehel conjecture on potentially $P_{k}$-graphic sequences is true. Science in China, Ser. A, 1998, 41, (1998) 5, pp. 510-520.

[18] J. Li, J. Yin, The smallest degree sum that yields potentially $K_{r, r}$-graphic sequences. Science in China, Ser. A, 45 (2002) 6, pp. 694-705.

[19] J. Li, J. Yin, An extremal problem on potentially $K_{r, s}$-graphic sequences. Discrete Math., 260 (2003), 295-305.

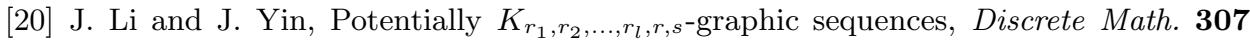
(2007), no. 9-10, 1167-1177.

[21] J. Li, J. Yin, Two sufficient conditions for a graphic sequence to have a realization with prescribed clique size. Discrete Math. 301 (2005), no. 2-3, 218-227.

[22] L.T. Ollmann, $K_{2,2}$-saturated graphs with a minimal number of edges. Proc. 3rd Southeast Conference on Combinatorics, Graph Theory and Computing, (1972) 367392.

[23] J. Schmitt, On Potentially P-graphic Degree Sequences and Saturated Graphs. Ph.D. Dissertation, Emory University. May 2005.

[24] Z. Tuza, $C_{4}$-saturated graphs of minimum size. Acta Universitatis Carolinae - Mathematica et Physica, 30 (1989) No. 2 161-167. 